\begin{tabular}{|c|l|}
\hline Title & $\begin{array}{l}\text { Synthesis of a tetrasaccharide repeating unit of O-antigenic poly saccharide of Sal monella enteritidis by use of unique } \\
\text { and odorless dodecyl thiogly cosyl donors }\end{array}$ \\
\hline Author(s) & Son, Sang-Hyun; Tano, Chiharu; Furuike, Tetsuy a; Sakairi, Nobuo \\
\hline Citation & $\begin{array}{l}\text { Tetrahedron Letters, 49(36), 5289-5292 } \\
\text { https://doi.org/10.1016/.tetlet.2008.06.097 }\end{array}$ \\
\hline Issue Date & 2008-09-01 \\
\hline Doc URL & http://hdl.handle.net/2115/38356 \\
\hline Type & article (author version) \\
\hline Additional Information & There are other files related to this item in HUSCAP. Check the above URL. \\
\hline File Information & TL2008.pdf () \\
\hline
\end{tabular}

Instructions for use 


\title{
Synthesis of tetrasaccharide repeating unit O-antigenic polysaccharide of Salmonella enteritidis by use of unique and odorless dodecyl thioglycosyl donors
}

\author{
Sang-Hyun Son ${ }^{\mathrm{a}}$, Chiharu Tano ${ }^{\mathrm{a}}$, Tetsuya Furuike ${ }^{\mathrm{b}}$, and Nobuo Sakairi ${ }^{\mathrm{a}, *}$ \\ ${ }^{a}$ Graduate School of Environmental Science, Hokkaido University, Kita-ku, Sapporo 060-0810, Japan \\ ${ }^{b}$ Department of Chemical and Materials Engineering, Faculty of Chemistry, Materials and Bioengineering, \\ and High Technology Research Center, Kansai University, Suita, Osaka 564-8680, Japan
}

\section{Abstract}

The first total synthesis of a unique tetrasaccharide repeating unit of lipopolysaccharide from Salmonella enteritidis has been accomplished by assembly of dodecyl thioglycosides. The crucial key steps were preparation of a rare branched dideoxy sugar, D-tyvelose (3,6-dideoxy-D-arabino-D-hexose) and sequential regioselective glycosylation at 2,3-positions of a central D-mannose residue 5 with D-tyvelose 6 and D-galactose donors 7.

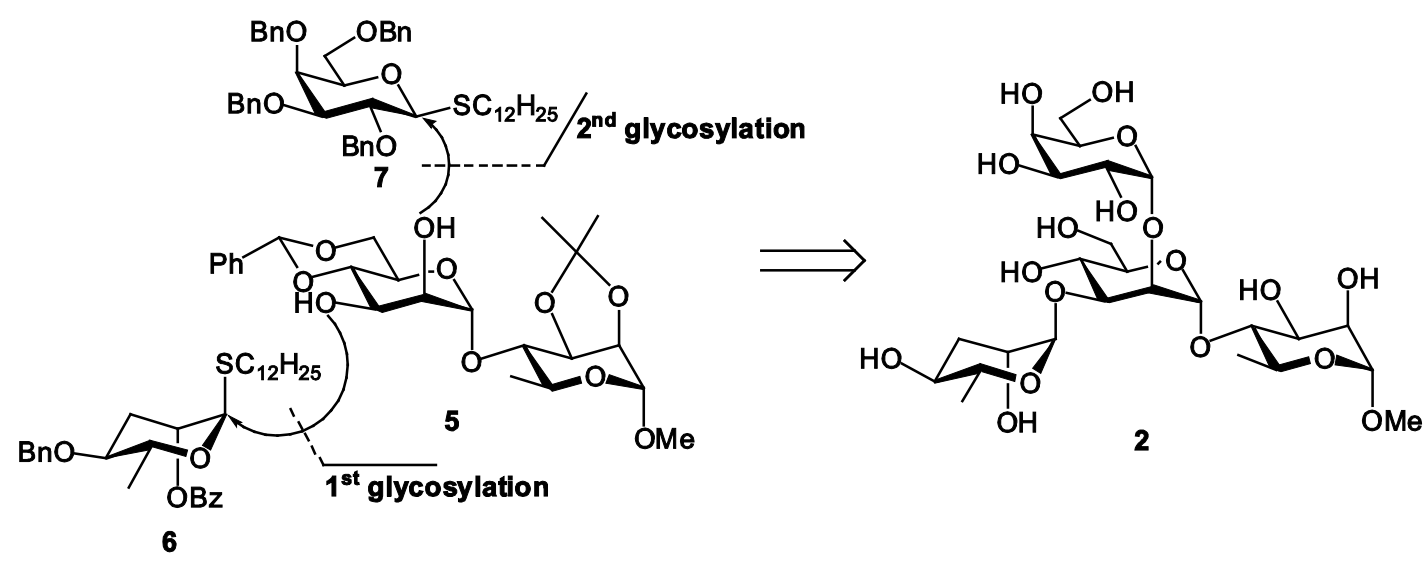


Salmonella enteritidis is known as a virulence food-borne enteric human pathogen, ${ }^{1}$ which colonize inert food contact surfaces to form biofilms. ${ }^{2}$ Like other Gram-negative bacteria, it has lipopolysaccharide (LPS), which consists of anchoring lipid A and $O$-antigenic polysaccharide, at the outer membrane. Isolation and characterization of its lipopolysaccharide (LPS), have been extensively studied, to understand the molecular mechanisms of Salmonella infection, adherence and biofilm formation on inert surfaces. ${ }^{3} \quad$ Recently, it was reported that $O$-antigen polysaccharide of Salmonella enteritidis has a repeating tetrasaccharide structure, $\rightarrow 3)-\alpha-D-G a l p-(1 \rightarrow 2)-[\alpha-D-T y v p-(1 \rightarrow 3)]-\alpha-D-M a n p-(1 \rightarrow 4)-\alpha-L-R h a p-\left(1 \rightarrow \quad(\mathbf{1}) .{ }^{4} \quad\right.$ Its unusual feature is the occurrence of a rare dideoxy sugar, D-tyvelose (3,6-dideoxy-D-arabino-D-hexose), which was deduced to play an important role in pathogenesis. $^{5} \quad$ Furthermore, central D-mannose residue in the repeating tetrasaccharide has three different monosaccharides at 1,2,3-positions. Due to its structural uniqueness and biomedical potential, we undertook to synthesize oligosaccharides related to $\mathbf{1}$. Herein, we report the first total synthesis of a methyl glycoside 2 of the repeating unit(see Fig. 1).

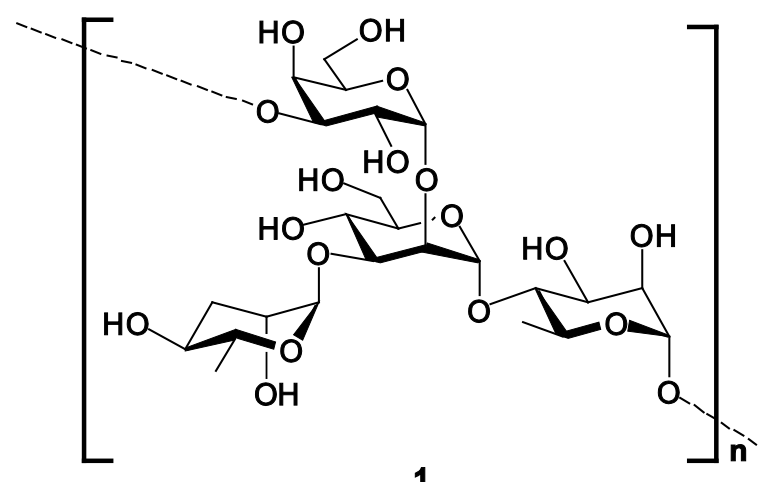

Figure 1. The structure of $O$-antigenic polysaccharide isolated from Salmonella enteritidis. 
Key problems needing to be addressed in the synthesis of $\mathbf{2}$ were preparation of a tyvelose donor and introduction of three monosaccharide moieties into the central mannose residue by efficient glycosylation methods. Retrosynthetic analysis shown in Scheme 1 suggested that sequential regioselective glycosylation of a disaccharide acceptor 5 having two free hydroxyl groups with D-tyvelose 6 and D-galactose donors 7 provides the target tetrasaccharide 2 . The sterically less hindered equatorial hydoxyl group at C-3' would be the first glycosylation site. Furthermore, $\mathbf{5}$ was to be synthesized from a mannosyl donor $\mathbf{4}$ and known methyl rhamnoside acceptor ${ }^{6} 3$. In all glycosylation reactions required for the construction of 2 , we planned to use our recently developed dodecyl thioglycosyl donor, ${ }^{7}$ of which advantage is that they are able to prepare under almost odorless conditions.

According to the synthetic plan mentioned above, we initiated to prepare three dodecyl thioglycoside donors 4, 6, 7 as depicted in Scheme 2. Saponification of the acetylated thiomannopyranoside $\mathrm{T}^{7 \mathrm{~d}} \mathbf{8}$ and subsequent $O$-benzylidenation and O-benzoylation affored the fully protected derivative 4 in $71 \%$ yield. For the construction of $\alpha$-mannosidic linkage, benzoly group with neighboring group participation was anticipated to be the best choice to protect the C2 hydroxyl group and it can be readily converted to free hydroxylic sites on the key intermediate $\mathbf{5}$ for the installation of the branched tyvelose moiety and galactose moiety. Moreover, $\mathbf{4}$ was also considered to be a precursor of the tyvelose donor $\mathbf{6}$. Thus the benzylidene acetal in 4 was regioselectively opened with $\mathrm{BH}_{3} \cdot \mathrm{Me}_{2} \mathrm{NH}-\mathrm{BF}_{3} \cdot \mathrm{Et}_{2} \mathrm{O}$ in dichloromethane furnished the primary alcohol ${ }^{8} \mathbf{9}$ in $82 \%$ yield. Next deoxygenation seemed to be a crucial step in our synthesis, because $\mathbf{9}$ has a reducible thioacetal function at the anomeric center. For deoxygenation of the primary alcohol, ${ }^{9} \mathbf{9}$ was subjected to 


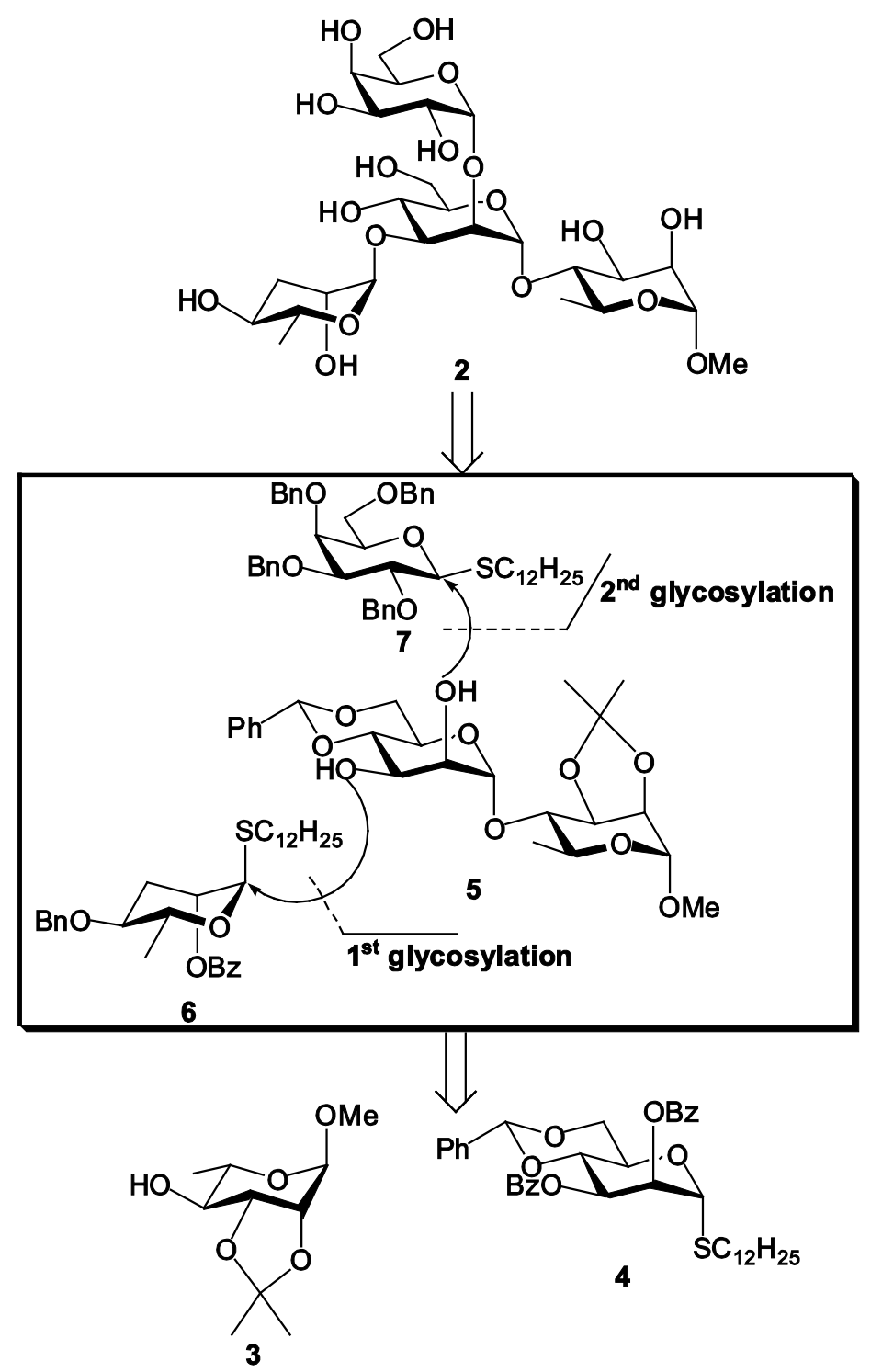

Scheme 1. Retrosynthetic analysis.

O-tosylation followed by reduction with $\mathrm{NaBH}_{4}$ in DMF to provide the dodecy thio D-rhamnoside $\mathbf{1 0}$ in a yield of $81 \%$. The second deoxygenation ${ }^{10}$ at C3 was performed after transformation into a monobenzoate $\mathbf{1 1}$ in three steps including de-O-benzoylation, formation of an orthoester, and its acid-catalyzed ring opening. The resulting the kinetically preferred axial benzolate $\mathbf{1 1}$ obtained in an overall yield of 
79\% was converted into thiocarbonylimidazole intermediate 12, which was subjected radical reduction with $\mathrm{Bu}_{3} \mathrm{SnH}^{10 \mathrm{~b}, \mathrm{c}}$ in tolutene to give the tyvelose donor 6 in $82 \%$ (see Scheme 3).
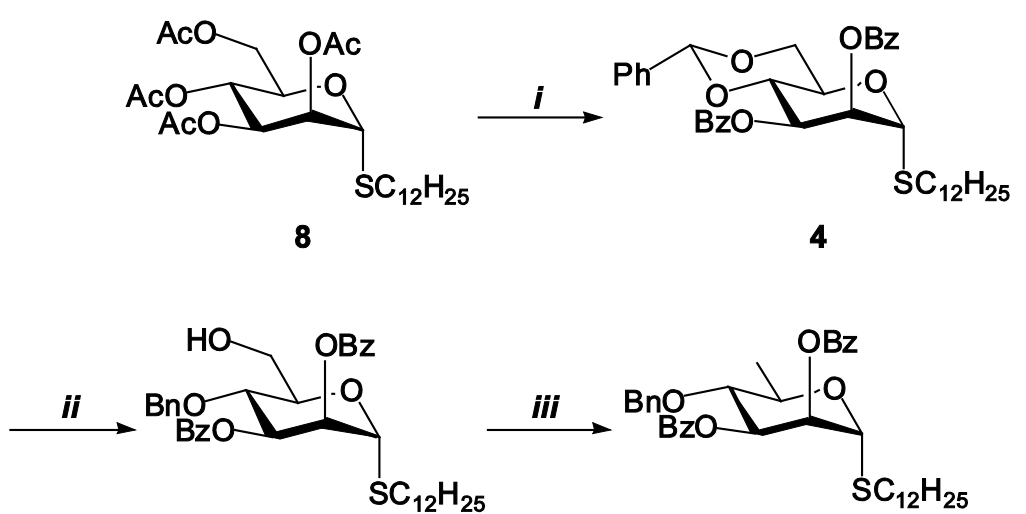

9

10

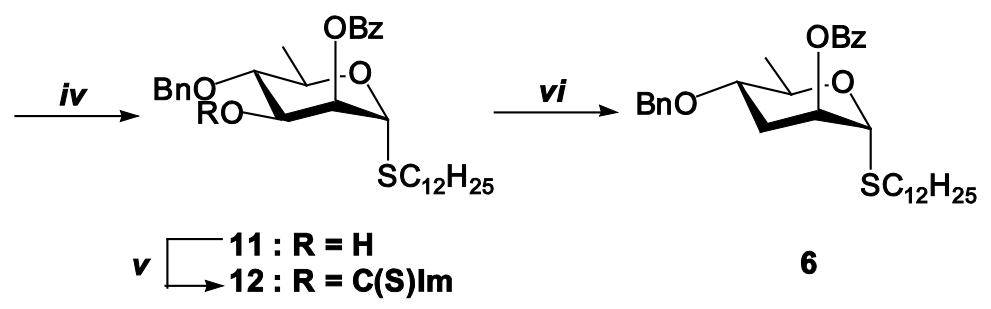

Scheme 2. Preparation of D-mannose and D-tyvelose donors. Reagents and conditions: (i) (a) $\mathrm{NaOMe} / \mathrm{MeOH}, \mathrm{rt}, 3$ h; (b) $\mathrm{PhCH}(\mathrm{OMe})_{2}$, cat. CSA, DMF, $50{ }^{\circ} \mathrm{C}, 4$ h; (c) $\mathrm{BzCl}, \mathrm{DMAP}$, pyridine, $50{ }^{\circ} \mathrm{C}, 3 \mathrm{~h}, 71 \%$ over 3 steps; (ii) $\mathrm{BH}_{3} \cdot \mathrm{Me}_{2} \mathrm{NH}, \mathrm{BF}_{3} \cdot \mathrm{Et}_{2} \mathrm{O}, \mathrm{CH}_{2} \mathrm{Cl}_{2}, 0{ }^{\circ} \mathrm{C}, 1 \mathrm{~h}, 82 \%$ (Ref. 8); (iii) (a) TsCl, DMAP, pyridine, $0{ }^{\circ} \mathrm{C}$ to $50{ }^{\circ} \mathrm{C}$, overnight; (b) $\mathrm{NaBH}_{4}$, DMF, $70{ }^{\circ} \mathrm{C}, 3 \mathrm{~h}$ 81\% over 2 steps (Ref. 9); (iv) (a) $\mathrm{NaOMe} / \mathrm{MeOH}-\mathrm{CH}_{2} \mathrm{Cl}_{2}$, rt, 3 h; (b) $\mathrm{PhC}(\mathrm{EtO})_{3}$, cat. CSA, $\mathrm{CH}_{2} \mathrm{Cl}_{2}$, rt, $3 \mathrm{~h}$; (c) $80 \% \mathrm{AcOH}$ in $\mathrm{H}_{2} \mathrm{O}, 80^{\circ} \mathrm{C}, 2$ h, $79 \%$ over 3steps (Ref. 10b and 10c); (v) $\mathrm{C}(\mathrm{S}) \mathrm{Im}_{2}$, imidazole, $\left(\mathrm{CH}_{2} \mathrm{Cl}\right)_{2}, 80{ }^{\circ} \mathrm{C}, 18 \mathrm{~h}, 98 \%$; (vi) $\mathrm{Bu}_{3} \mathrm{SnH}$, AIBN, toluene, reflux, $30 \mathrm{~min}$, 82\% (Ref.10). 

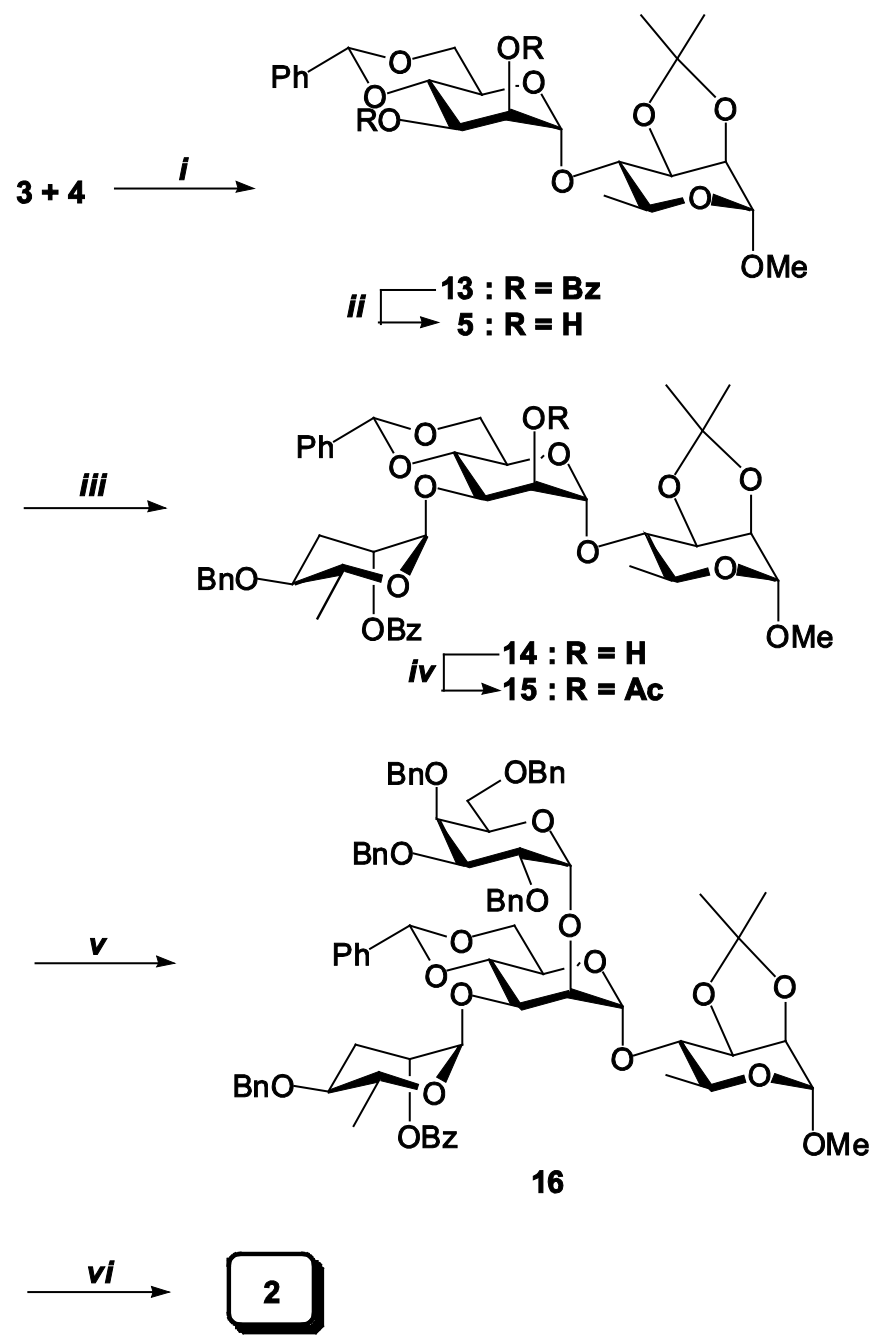

Scheme 3. Construction of tetrasaccharide 2 by assembly of dodecy thioglycosides. Reagents and conditions: (i) NIS, TfOH, $\mathrm{CH}_{2} \mathrm{Cl}_{2}, \mathrm{MS} 4 \AA$, $-20{ }^{\circ} \mathrm{C}$ to $0{ }^{\circ} \mathrm{C}, 30 \mathrm{~min}, 90 \%$; (ii) $\mathrm{NaOMe} / \mathrm{MeOH}-\mathrm{CH}_{2} \mathrm{Cl}_{2}$, rt, 3 h, 96\%; (iii) 6, NIS, TfOH, $\mathrm{CH}_{2} \mathrm{Cl}_{2}, \mathrm{MS} 4 \AA$, $-40{ }^{\circ} \mathrm{C}, 30 \mathrm{~min}$, 92\%; (iv) $\mathrm{Ac}_{2} \mathrm{O}$, pyridine; (v) 7, BSP, $\mathrm{Tf}_{2} \mathrm{O}, \mathrm{DTBM}, \mathrm{CH}_{2} \mathrm{Cl}_{2}, \mathrm{MS} 4 \AA$, $-78{ }^{\circ} \mathrm{C}, 72 \%$; (vi) (a) 80\% AcOH in $\mathrm{H}_{2} \mathrm{O}, 50{ }^{\circ} \mathrm{C}, 24$ h; (b) $\mathrm{NaOMe} / \mathrm{MeOH}$, rt, 6 h; (c) Pd/C, $\mathrm{MeOH}-\mathrm{H}_{2} \mathrm{O}-\mathrm{AcOH}, \mathrm{H}_{2}$, rt, 24 h, 76\% over three steps. 
Assembly of these thioglycosyl donors toward 2 was performed by use of thiophyllic reagents as the promoters. The first coupling of $\mathbf{3}$ and $\mathbf{4}$ proceeded at -20 ${ }^{\circ} \mathrm{C}$ to $0{ }^{\circ} \mathrm{C}$ in the presence of molecular sieves and a promoter, $N$-iodosuccinimide and catalytic triflic acid (NIS-TfOH), ${ }^{11}$ giving the disaccharide ${ }^{12} \mathbf{1 3}$ in $90 \%$ yield. Subsequently, two benzolyl groups at 2',3'-positions of $\mathbf{1 3}$ was removed by Zemplén method to give the disaccharide acceptor ${ }^{14} \mathbf{5}$ in $96 \%$ yield. Using this diol acceptor, we examined sequential and regioselective glycosylation in order to shorten the synthetic route to 2. For selective glycosylationat the equatorial hydroxyl group at 3'-position, we used excess amount of the diol acceptor 5 . Thus, a mixture of $5(0.25 \mathrm{mmol})$ and the tyvelose donor $6(0.125 \mathrm{mmol})$ was activated with NIS-TfOH at $-40{ }^{\circ} \mathrm{C}$. As a result, the desired trisaccharide ${ }^{12} \mathbf{1 4}$ was obtained with completed $\alpha$-stereoselectivity in excellent yield. The presence of the newly introduce $\alpha-(1 \rightarrow 3)$ linkage between D-tyvelose and D-mannose units in $\mathbf{1 4}$ was unambiguously ascertained by ${ }^{1} \mathrm{H}$ COSY NMR spectroscopy after conversion of the acetylated derivative ${ }^{12} \mathbf{1 5}$. Careful examination of the spectrum showed $\mathrm{H}-2$ protons for the mannose residue at lower magnetic field ( $\delta$ 5.21) as a double doublet which was assigned by a cross-peak between $\mathrm{H}-1$ and $\mathrm{H}-2$ protons. The regioselectivity is explainable by the facts that the equatorial 3'-hydroxyl group is less hindered than the axial 2'-hydroxyl group and that presence of bulky L-rhamnose residue at the anomeric position of the mannose residue reduce the reactivity of neighboring 2'-hydoxyl group. Furthermore, comparison of decoupling and non-decoupling HSQC spectra revealed signals of anomeric carbons at $\delta$ $99.0\left(J_{\mathrm{C} 1^{\prime}, \mathrm{H} 1^{\prime}}=175.9 \mathrm{~Hz}, \mathrm{C}-1^{\prime}\right), 97.8\left(J_{\mathrm{C} 1, \mathrm{H} 1}=170.0 \mathrm{~Hz}, \mathrm{C}-1\right)$, and $97.7\left(J_{\mathrm{C} 1^{\prime \prime}, \mathrm{H} 1^{\prime \prime}}=172.9\right.$ Hz, C-1"), suggesting 15 had all $\alpha$-glycosidic linkages. The next coupling between the donor $\mathbf{7}$ and the trisaccharide $\mathbf{1 4}$ was performed using a powerful promoters of 
thioglycosides so far reported, because of the prediction of the extremely poor reactivity of 14. Thus, the donor $\mathbf{7}$ was pre-activated with the 1-benzenesulfinyl piperidine and triflic anhydride $\left(\mathrm{BSP}-\mathrm{Tf}_{2} \mathrm{O}\right)^{12}$ combination in the presence of 2,6-di-tert-butyl-4-methylpyridine (DTBP) in dichloromethane at $-78{ }^{\circ} \mathrm{C}$ and then treated with the acceptor $\mathbf{1 4}$. Interestingly, desired tetrasaccharide ${ }^{12} \mathbf{1 6}$ was isolated in 72\% yield with complete $\alpha$-stereoselectivity. No trace of the $\beta$-anomer was detected by both chromatographic and NMR spectroscopic analyses of the crude reaction mixture. Similar results had been obtained previously with fully benzylated thiogalactosyl donors using sulfonium triflate per-activation procedure. ${ }^{14}$

Finally, global deprotection of $\mathbf{1 6}$ was successfully carried out by a three-step procedure that involved the hydrolysis of the acetal protecting groups by treatment with aqueous $\mathrm{AcOH}$, transesterification of the $O$-benzoyl groups with sodium methoxide in methanol, and catalytic hydrogenolysis over Pd/C to remove the $O$-benzyl groups. The unprotected tetrasaccharide ${ }^{12} 2$ thus obtained was characterized spectroscopic analyses. Representative ${ }^{1} \mathrm{H}$ and ${ }^{13} \mathrm{C}$ NMR chemical shift data of the synthetic tetrasaccharide 2 is excellent agreement with the reported data ${ }^{4}$ of the natural polysaccharide $\mathbf{1}$.

In conclusion, total synthesis of a tetrasaccharide repeating unit of $O$-antigenic polysaccharide of Salmonella enteritidis was achieved by assembly of dodecyl thioglycosyl donors. Our finding such as regioselective and sequential glycosylation 2,3-positions of core mannose residue would make it possible to provide easily various oligosaccharides analogous of $\mathbf{2}$, which are useful substrates for biological examinations towards its infection control. 


\section{Reference and notes}

1. (a) Dhir, V. K.; Dodd, C. E. Appl. Environ. Microbiol. 1995, 61, 1731-1738; (b) Jones, K.; Bradshaw, S. B. J. Appl. Bacteriol. 1996, 80, 458-464.

2. Austin, J. W.; Sanders, G.; Kay, W. W.; Collinson, S. K. FEMS Microbiol. Lett. 1998, 162, 295-301.

3. (a) Carpentier, B.; Cerf, O. J. Appl. Bacteriol. 1993, 75, 499-511; (b) Austin, J. W.; Bergeron, G. J. Dairy Res. 1995, 62, 509-519.

4. Snyder, D. S.; Gibson, D.; Heiss, C.; Kay, W.; Azadi, P. Carbohydr. Res. 2006, 341, $2388-2397$.

5. (a) Appleton, J. A.; Schain, L. R.; McGregor, D. D. Immunology 1988, 65, 487-492;

(b) Denkers, E. Y.; Hayes, C. E.; Wassom, D. L. Exp. Parasitol. 1991, 72, 403-410;

(c) Nitz, M.; Bundle D. R. J. Org. Chem. 2000, 65, 3064-3073; (d) Hirooka, M.;

Yoshimura, A.; Saito, I.; Ikawa, F.; Uemoto, Y.; Koto, S.; Takabatake, A.; Taniguchi, A.; Shinoda, Y.; Morinaga, A. Bull. Chem. Soc. Jpn. 2003, 76, 1409-1421 and references there in.

6. Davis, B.; Brandstetter, T. W.; Smith, C.; Hackett, L.; Winchester, B. G.; Fleet, G. W. J. Tetrahedron Lett. 1995, 36, 7505-7510.

7. (a) Matsui, H.; Furukawa, J.; Awano, T., Nishi, N.; Sakairi, N. Chem. Lett. 2000, 326-327; (b) Matsuoka, K.; Onaga, T.; Mori, T.; Sakamoto, J. -I.; Koyama, T.; Sakairi, N.; Hatano, K.; Terunuma, D. Tetrahedron Lett. 2004, 45, 9383-9386; (c) Son, S. -H.; Tano, C.; Furukawa, J. -i.; Furuike, T.; Sakairi, N. Org. Biomol. Chem. 2008, 6, 1441-1449.

8. Oikawa, M.; Liu, W. -C.; Nakai, Y.; Koshida, S.; Fukase, K.; Kusumoto, S. Synlett 1996, $1179-1180$. 
9. Van den Bos, L. J.; Boltje, T. J.; Provoost, T.; Mazurek, J.; Overkleeft, H. S.; van der Marel, G. A. Tetrahedron Lett. 2007, 48, 2697-2700.

10.(a) Rasmussen, J. R. J. Org. Chem. 1980, 45, 2725-2727; (b) Bart, R.; Pavol, K. Synthesis 2004, 2505-2508; (c) Turek, D.; Sundgren, A.; Lahmann, M.; Oscarson, S. Org. Biomol. Chem. 2006, 4, 1236-1241.

11.Veeneman, G. H.; van Leeuwen, S. H.; van Boom, J. H. Tetrahedron Lett. 1990, 31, 1331-1334.

12. Representative ${ }^{1} \mathrm{H}$ NMR, and other physical data of compound 13, 5, 14, 15, 16, and 2. Compound 13: $[\alpha]_{\mathrm{D}}{ }^{20.0}-94.3\left(c\right.$ 1.00, $\left.\mathrm{CHCl}_{3}\right) ;{ }^{1} \mathrm{H}$ NMR (300 MHz, $\left.\mathrm{CDCl}_{3}\right): \delta 5.81$ $\left(\mathrm{dd}, 1 \mathrm{H}, J_{3^{\prime}, 4^{\prime}}=3.4 \mathrm{~Hz}, \mathrm{H}-3^{\prime}\right), 5.65\left(\mathrm{dd}, 1 \mathrm{H}, J_{2^{\prime}, 3^{\prime}}=3.5 \mathrm{~Hz}, \mathrm{H}-2^{\prime}\right), 5.12\left(\mathrm{~s}, 1 \mathrm{H}, \mathrm{H}-1^{\prime}\right)$, 4.88 (s, $1 \mathrm{H}, \mathrm{H}-1), 1.37$ (d, 3H, $J=6.1 \mathrm{~Hz}, \mathrm{H}-6)$; HRMS (FAB) calcd for $\mathrm{C}_{37} \mathrm{H}_{41} \mathrm{O}_{12}$ $[\mathrm{M}+\mathrm{H}]^{+}:$677.2593, found 677.2592.

Compound 5: $[\alpha]_{\mathrm{D}}{ }^{20.0}-43.9\left(c\right.$ 1.00, $\left.\mathrm{CHCl}_{3}\right) ;{ }^{1} \mathrm{H}$ NMR (300 MHz, $\left.\mathrm{CDCl}_{3}\right): \delta 4.90$ (s, 1H, H-1'), 4.85 (s, 1H, H-1), 1.27 (d, 1H, $J=6.3 \mathrm{~Hz}, \mathrm{H}-6)$; HRMS (FAB) calcd for $\mathrm{C}_{23} \mathrm{H}_{33} \mathrm{O}_{10}[\mathrm{M}+\mathrm{H}]^{+}:$469.2068, found 469.2075.

Compound 14: $[\alpha]_{\mathrm{D}}^{21.8}+38.6\left(c\right.$ 1.00, $\left.\mathrm{CHCl}_{3}\right) ;{ }^{1} \mathrm{H} \mathrm{NMR}\left(600 \mathrm{MHz}, \mathrm{CDCl}_{3}\right): \delta$ 8.01-7.18 (m, 15H, CHarom), 5.59 (s, 1H, PhCH), 5.31 (s, 1H, H-2"), 5.28 (s, 1H, H-1"), 4.95 (s, 1H, H-1'), 4.84 (s, 1H, H-1), 4.62 (d, 1H, $J=11.0$ Hz, PhCH $H_{2}$ ), 4.47 (d, $1 \mathrm{H}, J=11.6 \mathrm{~Hz}, \mathrm{PhCH}_{2}$ ), 4.31-4.28 (m, 1H, H-6'a), 4.25-4.18 (m, 1H, H-6'b), 4.16-4.02 (m, 5H, H-2, 3, 2', 3', 4'), 3.89-3.85 (m, 1H, H-5'), 3.84-3.78 (m, 1H, H-5'), 3.69-3.62 (m, 1H, H-5), 3.49-3.44 (m, 1H, H-4"), 3.58-3.42 (m, 1H, H-4), 3.36 (s, 3H, OMe), 2.53 (s, 1H, OH), 2.40-2.34 (m, 1H, H-3"), 2.04-1.96 (m, 1H, H-3"), 1.52 (s, 3H, CH $\left.H_{3}\right), 1.35$ (d, 3H, $\left.J=6.6 \mathrm{~Hz}, \mathrm{H}-6 "\right), 1.33$ (s, 3H, CH $\left.\mathrm{CH}_{3}\right), 1.27$ (d, 
$3 \mathrm{H}, J=6.6 \mathrm{~Hz}, \mathrm{H}-6)$; HRMS (FAB) calcd for $\mathrm{C}_{43} \mathrm{H}_{53} \mathrm{O}_{14}[\mathrm{M}+\mathrm{H}]^{+}:$793.3430, found 793.3437.

Compound 15: ${ }^{1} \mathrm{H}$ NMR (300 MHz, $\mathrm{CDCl}_{3}$ ): $\delta$ 7.97-7.22 (m, 15H, CHarom), 5.63 (s, 1H, PhCH), 5.26 (brd, $1 \mathrm{H}, \mathrm{H}-2^{\prime \prime}$ ), 5.21 (dd, $1 \mathrm{H}, J_{2^{\prime}, 3^{\prime}}=3.5 \mathrm{~Hz}, \mathrm{H}-2^{\prime}$ ), 5.16 (s, $1 \mathrm{H}$, H-1"), 4.91 (d, 1H, $\left.J_{1^{\prime}, 2^{\prime}}=1.2 \mathrm{~Hz}, \mathrm{H}-1^{\prime}\right), 4.85$ (s, 1H, H-1), $4.62(\mathrm{~d}, 1 \mathrm{H}, J=11.6 \mathrm{~Hz}$, $\mathrm{PhCH}_{2}$ ), 4.47 (d, 1H, $J=11.6 \mathrm{~Hz}, \mathrm{PhCH}_{2}$ ), 4.34 (dd, 1H, $\left.J_{3^{\prime}, 4^{\prime}}=9.5 \mathrm{~Hz}, \mathrm{H}-3^{\prime}\right), 4.27$ (dd, $1 \mathrm{H}, J_{5^{\prime}, 6^{\prime} \mathrm{b}}=4.2 \mathrm{~Hz}, \mathrm{H}-6$ 'b), 4.18-4.06 (m, 4H, H-2, 3, 4', 6'a), 3.87-3.76 (m, 2H, H-5', 5"), 3.70-3.61 (m, 1H, H-5), 3.55-3.33 (m, 2H, H-4, 4"), 3.35 (s, 3H, OMe), 2.33-2.26 (m, 1H, H-3"), 2.18 (s, 3H, acetyl), 2.08-1.92 (m, 1H, H-3"), 1.51 (s, 3H, $\mathrm{CH}_{3}$ ), 1.33 (s, 3H, $\mathrm{CH}_{3}$ ), 1.32 (d, 3H, $J=6.3 \mathrm{~Hz}, \mathrm{H}-6^{\prime \prime}$ ), 1.31 (d, 3H, $J=6.3 \mathrm{~Hz}$, H-6); ${ }^{13} \mathrm{C}$ NMR (150.9 MHz, $\left.\mathrm{CDCl}_{3}\right): \delta 170.3,165.4,138.2,137.3,133.2,130.1$, $129.8,128.7,128.4,128.4,128.1,127.9,127.7,126.0,109.2,101.3,99.0\left(J_{\mathrm{C} 1^{\prime}, \mathrm{H} 1^{\prime}}=\right.$ $\left.175.9 \mathrm{~Hz}, \mathrm{C}-1^{\prime}\right), 97.8\left(J_{\mathrm{C} 1, \mathrm{H} 1}=170.0 \mathrm{~Hz}, \mathrm{C}-1\right), 97.7\left(J_{\mathrm{C} 1^{\prime \prime}, \mathrm{H} 1^{\prime \prime}}=172.9 \mathrm{~Hz}, \mathrm{C}-1^{\prime \prime}\right), 81.2$, 78.7, 76.7, 76.0, 74.9, 72.1, 71.4, 71.0, 70.8, 68.8, 68.7, 64.6, 63.8, 54.9, 29.3, 28.1, 26.4, 21.0, 18.2, 17.4 .

Compound 16: $[\alpha]_{\mathrm{D}}^{21.0}+34.1\left(\right.$ c $\left.1.00, \mathrm{CHCl}_{3}\right) ;{ }^{1} \mathrm{H}$ NMR $\left(600 \mathrm{MHz}, \mathrm{CDCl}_{3}\right): \delta 5.62$ (d, 1H, $\left.J_{1^{\prime \prime \prime}, 2^{\prime \prime \prime}}=3.8 \mathrm{~Hz}, \mathrm{H}-1^{\prime \prime \prime}\right), 5.29$ (s, 1H, H-1"), 4.93 (s, 1H, H-1'), 4.83 (s, 1H, H-1), 4.39 (dd, 1H, $\left.J_{3^{\prime}, 4^{\prime}}=10.4 \mathrm{~Hz}, \mathrm{H}-3^{\prime}\right), 4.17\left(\mathrm{dd}, 1 \mathrm{H}, J_{2^{\prime}}, 3^{\prime}=5.0 \mathrm{~Hz}, \mathrm{H}-2^{\prime}\right)$, 2.29-2.23 (m, 1H, H-3"), 1.90-1.84 (m, 1H, H-3"), 1.32 (d, 3H, J = 6.1 Hz, H-6"'), $1.18(\mathrm{~d}, 3 \mathrm{H}, \mathrm{J}=6.1 \mathrm{~Hz}, \mathrm{H}-6)$; HRMS (FAB) calcd for $\mathrm{C}_{77} \mathrm{H}_{87} \mathrm{O}_{19}[\mathrm{M}+\mathrm{H}]^{+}$: 1315.5836, found 1315.5829.

Compound 2: ${ }^{1} \mathrm{H}$ NMR (300 MHz, $\mathrm{D}_{2} \mathrm{O}, 333 \mathrm{~K}$ ): (selected data) $\delta 5.25$ (d, $1 \mathrm{H}, J_{1^{\prime}, 2^{\prime}}$ = $\left.1.5 \mathrm{~Hz}, \mathrm{H}-1^{\prime}\right), 5.21$ (d, 1H, $\left.J_{1^{\prime \prime \prime}, 2^{\prime \prime \prime}}=3.7 \mathrm{~Hz}, \mathrm{H}-1^{\prime \prime \prime}\right), 4.90$ (brd, 1H, H-1), 4.70 (d, 1H, $\left.J_{1^{\prime \prime}, 2^{\prime \prime}}=1.4 \mathrm{~Hz}, \mathrm{H}-1^{\prime \prime}\right), 4.18-3.52(\mathrm{~m}, 29 \mathrm{H}), 3.40$ (s, 3H, OMe), 2.08-2.01 (m, 1H, 
H-3"), 1.90-1.78 (m, 1H, H-3"), 1.34 (d, 3H, J5, $6=6.4 \mathrm{~Hz}, \mathrm{H}-6), 1.28$ (d, 3H, $J_{5^{\prime}, 6^{\prime}}=$ $\left.6.2 \mathrm{~Hz}, \mathrm{H}-6{ }^{\prime}\right) ;{ }^{13} \mathrm{C}$ NMR (75 MHz, $\left.\mathrm{D}_{2} \mathrm{O}\right): \delta$ 104.1, 104.0, 103.5, 102.5, 84.4, 81.8, 80.1, 76.3, 74.3, 73.2, 73.1, 72.1, 72.1, 71.5, 70.2, 70.2, 70.0, 69.1, 64.0, 63.3, 57.6, 19.9, 19.6; HRMS (ESI) calcd for $\mathrm{C}_{25} \mathrm{H}_{44} \mathrm{O}_{18}\left[\mathrm{M}-\mathrm{H}^{+}\right]^{-}:$631.2455, found 631.2452. 13.(a) Crich, D.; Smith, M. Org. Lett. 2000, 2, 4067-4069; (b) Crich, D.; Smith, M. J. Am. Chem. Soc. 2001, 123, 9015-9020; (c) Crich, D.; Smith, M. J. Am. Chem. Soc. 2002, 124, 8867-8869; (d) Crich, D.; Lim, L. B. L. Org. React. 2004, 64, 115-251; (e) Crich, D.; Banerjee, A.; Li, W.; Yao, Q. J. Carbohydr. Chem. 2005, 24, 415-424.

14.(a) Codée, J. D. C.; Litjens, R. E. J. N.; den Heeten, R.; Overkleeft, H. S.; van Boom, J. H.; van der Marel, G. A. Org. Lett. 2003, 5, 1519-1522; (b) Codée, J. D. C.; van den Bos, L. J.; Litjens, R. E. J. N.; Overkleeft, H. S.; van Boom, J. H.; van der Marel, G. A. Org. Lett. 2003, 5, 1947-1950; (c) Wang, Y.; Huang, X.; Zhang, L. -H.; Ye, X.-S. Org. Lett. 2004, 6, 4415-4417; (d) Wang, C.; Wang, H.; Huang, X.; Zhang, L. -H.; Ye, X.-S. Synlett 2006, 2846-2850. 